\title{
Rotational thromboelastometry-guided blood product management in major spine surgery
}

\author{
Bhiken I. Naik, MBBCh,1,2 Thomas N. Pajewski, MD, PhD,,2 David L. Bogdonoff, MD,1 \\ Zhiyi Zuo, MD, PhD, ${ }^{1,2}$ Pamela Clark, MD, JD, ${ }^{3}$ Abdullah S. Terkawi, MD, ${ }^{1}$ \\ Marcel E. Durieux, MD, PhD, ${ }^{1,2}$ Christopher I. Shaffrey, MD, ${ }^{2}$ and Edward C. Nemergut, MD ${ }^{1,2}$ \\ Departments of ${ }^{1}$ Anesthesiology, ${ }^{2}$ Neurosurgery, and ${ }^{3}$ Pathology, University of Virginia, Charlottesville, Virginia
}

OBJECT Major spinal surgery in adult patients is often associated with significant intraoperative blood loss. Rotational thromboelastometry (ROTEM) is a functional viscoelastometric method for real-time hemostasis testing. In this study, the authors sought to characterize the coagulation abnormalities encountered in spine surgery and determine whether a ROTEM-guided, protocol-based approach to transfusion reduced blood loss and blood product use and cost.

METHODS A hospital database was used to identify patients who had undergone adult deformity correction spine surgery with ROTEM-guided therapy. All patients who received ROTEM-guided therapy (ROTEM group) were matched with historical cohorts whose coagulation status had not been evaluated with ROTEM but who were treated using a conventional clinical and point-of-care laboratory approach to transfusion (Conventional group). Both groups were subdivided into 2 groups based on whether they had received intraoperative tranexamic acid (TXA), the only coagulationmodifying medication administered intraoperatively during the study period. In the ROTEM group, 26 patients received TXA (ROTEM-TXA group) and 24 did not (ROTEM-nonTXA group). Demographic, surgical, laboratory, and perioperative transfusion data were recorded. Data were analyzed by rank permutation test, adapted for the 1:2 ROTEM-to-Conventional matching structure, with $p<0.05$ considered significant.

RESULTS Comparison of the 2 groups in which TXA was used showed significantly less fresh-frozen plasma (FFP) use in the ROTEM-TXA group than in the Conventional-TXA group (median 0 units [range 0-4 units] vs 2.5 units [range $0-13$ units], $p<0.0002$ ) but significantly more cryoprecipitate use (median 1 unit [range 0-4 units] in the ROTEM-TXA group vs 0 units [range $0-2$ units] in the Conventional-TXA group, $p<0.05$ ), with a nonsignificant reduction in blood loss (median 2.6 L [range 0.9-5.4 L] in the ROTEM-TXA group vs 2.9 L [0.7-7.0 L] in the Conventional-TXA group, $p=0.21$ ). In the 2 groups in which TXA was not used, the ROTEM-nonTXA group showed significantly less blood loss than the Conventional-nonTXA group (median $1 \mathrm{~L}$ [range $0.2-6.0 \mathrm{~L}$ ] vs $1.5 \mathrm{~L}$ [range $1.0-4.5 \mathrm{~L}$ ], $p=0.0005$ ), with a trend toward less transfusion of packed red blood cells ( $p R B C$ ) (median 0 units [range 0-4 units] vs 1 unit [range 0-9 units], $p=0.09$ ]. Cryoprecipitate use was increased and FFP use decreased in response to ROTEM analysis identifying hypofibrinogenemia as a major contributor to ongoing coagulopathy.

CONCLUSIONS In major spine surgery, ROTEM-guided transfusion allows for standardization of transfusion practices and early identification and treatment of hypofibrinogenemia. Hypofibrinogenemia is an important cause of the coagulopathy encountered during these procedures and aggressive management of this complication is associated with less intraoperative blood loss, reduced transfusion requirements, and decreased transfusion-related cost.

http://thejns.org/doi/abs/10.3171/2014.12.SPINE14620

KEY WORDS rotational thromboelastometry; spine surgery; hypofibrinogenemia; bleeding

ABBREVIATIONS ASA = American Society of Anesthesiologists; $\mathrm{A} 10=$ amplitude at 10 minutes; $\mathrm{A} 20=$ amplitude at 20 minutes; $\mathrm{CFT}=\mathrm{clot}$ formation time; $\mathrm{CT}=\mathrm{clotting}$ time; EXTEM = external temogram; FFP = fresh-frozen plasma; FIBTEM = fibrinogen temogram; INR = international normalized ratio; $M C F=$ maximum clot firmness; $M L=$ maximum lysis; $\mathrm{POC}=$ point of care; $\mathrm{POD}=$ postoperative day; $\mathrm{PRBC}=$ packed red blood cells; $\mathrm{PSO}=$ pedicle subtraction osteotomy; $\mathrm{PT}=$ prothrombin time; $\mathrm{PTT}=$ partial thromboplastin time; SPO = Smith-Petersen osteotomy; TXA = tranexamic acid.

SUBMITTED June 20, 2014. ACCEPTED December 3, 2014.

INCLUDE WHEN CITING Published online May 22, 2015; DOI: 10.3171/2014.12.SPINE14620.

DISCLOSURE Dr. Shaffrey reports a consultant relationship with Biomet, Globus, Medtronic, NuVasive, and Stryker; direct stock ownership in NuVasive; and holding patents for and receiving royalties from Biomet, Medtronic, and NuVasive. 
$\mathrm{M}$ AJOR deformity correction spine surgery can be associated with significant perioperative blood loss. ${ }^{12,16}$ This blood loss is related to multiple factors, including the number of surgically treated levels and osteotomies performed, primary versus repeat surgery, single versus staged procedures, and whether an anterior and/or posterior approach is performed. The risk of significant bleeding is further exacerbated in older patients because of a thin periosteum and wide vascular channels encountered in osteoporotic bone. ${ }^{9}$ Norton et al., in a retrospective review of 423 adult patients, reported major intraoperative blood loss (> $4 \mathrm{~L}$ ) in $24 \%$ of patients undergoing 3-column resection osteotomies. ${ }^{17}$ In a study of both pediatric and adult cases, Horlocker et al. demonstrated a $16 \%$ incidence of significant intraoperative bleeding, with the range of blood loss varying between 0.9 and $20 \mathrm{~L} .{ }^{8}$ Rapid, real-time evaluation of the coagulation system is paramount during ongoing major blood loss. Rotational thromboelastometry (ROTEM, TEM International $\mathrm{GmbH}$ ) is a rapid viscoelastometric method for hemostasis testing in whole blood. The interaction between coagulation factors and cellular components during the coagulation and subsequent lytic phase can be evaluated and reported in real time. By utilizing this dynamic functional hemostatic test, abnormalities of the different coagulation pathways such as platelet adhesion/aggregation, coagulation, clot strength, and fibrinolysis can be identified with minimal delay and treated with factor-specific therapy. In multiple studies, thromboelastometry use has been demonstrated to improve profiling of coagulation deficiencies and reduce blood product utilization. However, the majority of these studies have been restricted to cardiac, trauma, and major visceral surgery. $5,18,23$

In this study we hypothesized that use of ROTEM to guide transfusion can identify specific coagulation abnormalities and reduce blood product use and cost in major spine surgery.

\section{Methods}

\section{Data Source}

This study was approved by the University of Virginia Institutional Review Board for Health Sciences Research. The requirement for patient informed consent was waived. We performed a retrospective analysis of all cases in which patients had undergone major spine surgery with intraoperative ROTEM-guided therapy during a 1-year period (June 2012-July 2013). Historical controls were identified through the University of Virginia's Clinical Data Repository, which maintains demographic, financial, procedural, and length-of-stay data. Current Procedural Terminology (CPT) codes were used to identify potential patients in the database who had undergone major cervical, thoracic, and/or lumbar spine surgery with instrumentation. Thereafter a manual chart review for each identified patient was performed to ensure that the surgical procedure matched the CPT codes.

\section{Matching Procedure and Group Definitions}

Patients who had received ROTEM-guided therapy (ROTEM group) were matched (by surgical site [cervical vs thoracic vs lumbar], number of surgically treated vertebral levels, and osteotomies and interbody procedures performed) with historical cohorts whose coagulation status had not been evaluated with ROTEM but who were treated using a conventional clinical and point-of-care laboratory approach to transfusion (Conventional group). The only coagulation-modifying medication administered intraoperatively during the study period was tranexamic acid (TXA). TXA was administered at the discretion of the attending surgeon and anesthesiologist when significant blood loss was expected. A predefined expected blood loss for intraoperative TXA administration was not set, but in all cases blood loss of more than $1 \mathrm{~L}$ was anticipated. Contraindications for TXA administration included previous ischemic stroke, chronic kidney disease, and preexisting color vision changes. To reduce the confounding effect of TXA administration on intraoperative blood loss, the ROTEM group was further divided into 2 subgroups: patients who received only ROTEM-guided therapy (ROTEM-nonTXA group) and those who received ROTEM-guided therapy and TXA (ROTEM-TXA group). The patients in the ROTEM-TXA and ROTEMnonTXA groups were matched, as described above, with historical controls who were treated with a conventional approach to the management of intraoperative blood loss and either received TXA (Conventional-TXA group) or did not receive TXA (Conventional-nonTXA group).

After matching, perioperative data were collected using the electronic health record. Preoperative demographic data included sex, age, American Society of Anesthesiologists (ASA) physical status, body mass index, and preoperative coagulation parameters. Intraoperative data included TXA dose and units of packed red blood cells (pRBC), fresh-frozen plasma (FFP), and cryoprecipitate administered. Total volume of crystalloid, colloid, autologous blood transfusion, and blood loss was recorded. Postoperative variables included coagulation parameters for the day of surgery and postoperative day (POD) 1 and blood and blood products administered.

\section{Transfusion Algorithms}

In the conventionally treated patients (ConventionalTXA and Conventional-nonTXA groups) intraoperative transfusion therapy was directed by the results of laboratory and point-of-care (POC) testing as well as clinical observation (Fig. 1). Clinically guided therapy was based on a subjective assessment of the quality of clot in the surgical field by the attending surgeon and anesthesiologist. POC testing was performed in the blood bank and included prothrombin time (PT), international normalized ratio (INR), and platelet counts. Intraoperative fibrinogen levels were obtained by standard laboratory test. The frequency of POC and formal laboratory testing was at the discretion of the anesthesiology team.

In the patients receiving ROTEM-guided therapy (ROTEM-TXA and ROTEM-nonTXA groups), functional assessment of the coagulation profile was performed utilizing the EXTEM (external temogram) and FIBTEM (fibrinogen temogram) assays. A predefined treatment algorithm was used to manage the temogram abnormalities (Fig. 2). Variables reported from these assays included 


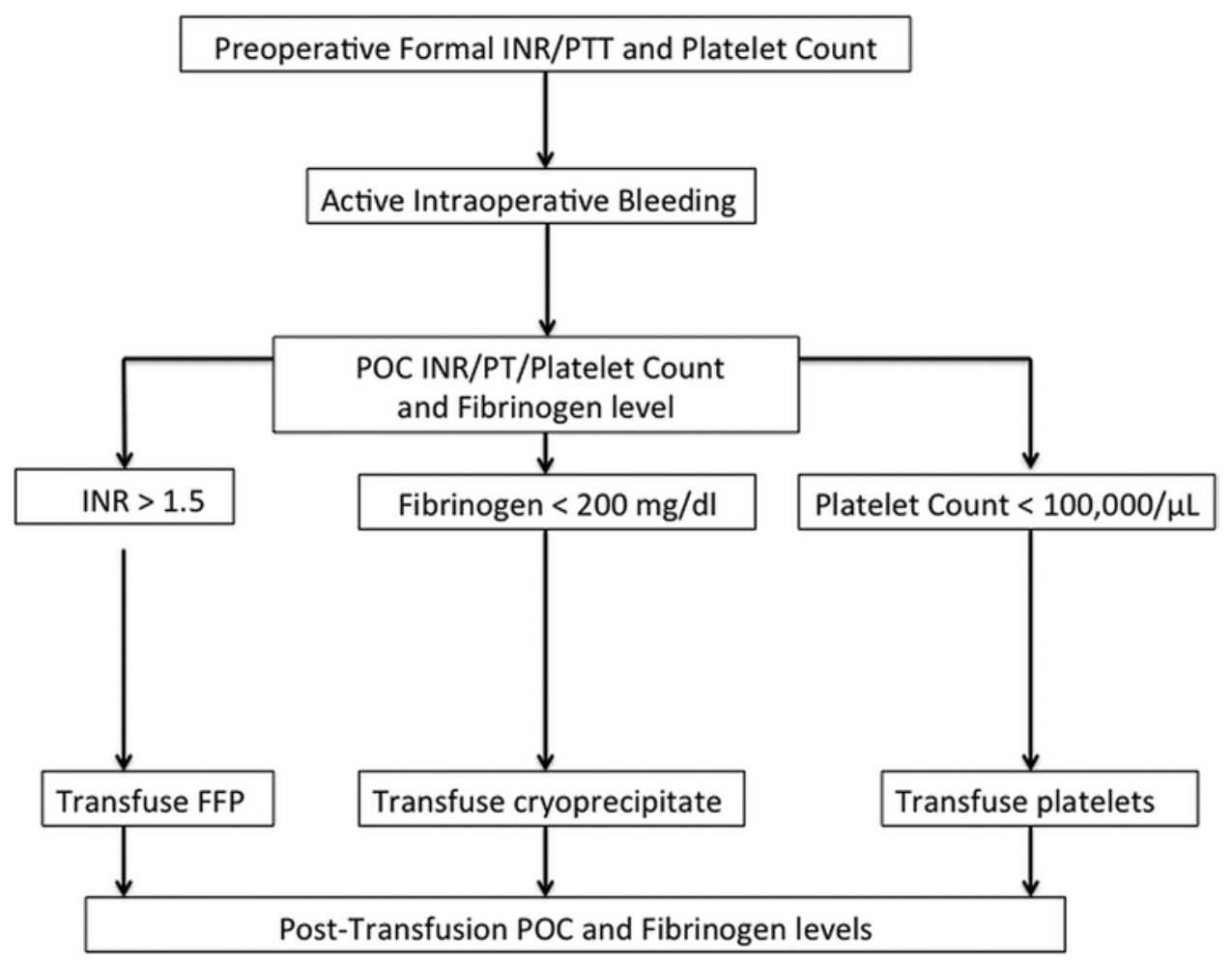

FIG. 1. Transfusion algorithm used in the historical controls (Conventional-TXA group and Conventional-nonTXA group). FFP = freshfrozen plasma; INR = international normalized ratio; $\mathrm{POC}=$ point of care; $\mathrm{PT}=$ prothrombin time; $\mathrm{PTT}=$ partial thromboplastin time.

clotting time (CT), clot formation time (CFT), $\alpha$ angle, amplitude at 10 minutes (A10), amplitude at 20 minutes (A20), maximum clot firmness (MCF), and maximum lysis (ML) (Figs. 3 and 4). ROTEM data were obtained after induction of anesthesia, after 1-2 units of blood loss, and at regular intervals thereafter at the discretion of the anesthesiologist. Prolongation of the CT (> 82 seconds) on the EXTEM is related to functionally low levels of factors II, VII, and X, which were treated with FFP. ${ }^{24}$ Low A10, A20, and MCF on the EXTEM are indicative of poor clot strength. This is related to either a platelet abnormality or a low fibrinogen level. The simultaneously assessed FIBTEM was used to further delineate whether a primary platelet or fibrinogen abnormality was responsible for the low EXTEM MCF. A low EXTEM MCF $(<52 \mathrm{~mm})$ in combination with a normal FIBTEM MCF (7-24 mm) indicates a platelet abnormality, which was treated with a platelet transfusion (Figs. 5 and 6). A low EXTEM MCF $(<52 \mathrm{~mm})$ and FIBTEM MCF $(<7 \mathrm{~mm})$ with a normal POC platelet count was treated with cryoprecipitate (Figs. 7 and 8). Packed red blood cells (pRBC) were transfused to maintain a hemoglobin level above $9-10 \mathrm{~g} / \mathrm{dl}$, at the discretion of the attending anesthesiologist.

\section{Cost Analysis}

A cost analysis was performed by first determining the total number of specific blood products transfused in the ROTEM groups. An average of the specific blood product per case in the ROTEM groups was determined. The average specific blood component per case in the ROTEM groups was then multiplied by the total number of cases in the conventional group. The final result was multiplied with the hospital unit cost of the specific blood product to determine total cost of specific component therapy, if ROTEM had been applied in the Conventional groups. The 2011 National Blood and Utilization Survey Report was used to determine the unit cost of blood and blood products. ${ }^{25}$

\section{Statistical Analysis}

Data analysis was performed with SPSS (IBM Corp.) and $\mathrm{R}$ (The $\mathrm{R}$ Project, version 2.15.1). For continuous variables, normality was assessed using the KolmogorovSmirnov test. The Mann-Whitney U-test was used to compare non-normally distributed data, and normally distributed data were assessed by the Student t-test for equality of means. We compared each of the outcome variables in the data set between the ROTEM groups and the Conventional groups. For all variables a rank permutation test was used. The rank permutation test is similar to the Wilcoxon signed-rank test, except that it was adapted to the 1:2 ROTEM-to-Conventional matching structure. A 1-way ANOVA was used to compare the mean differences of all EXTEM and FIBTEM variables over time. A p $<0.05$ was considered significant.

\section{Results \\ Study Population}

Between June 2012 and July 2013, 26 patients had ROTEM-guided therapy with TXA and 24 patients had ROTEM-guided therapy without TXA. The ROTEMTXA group was matched to historical controls in a 1:1 ratio. In the ROTEM-nonTXA group, 20 patients were 


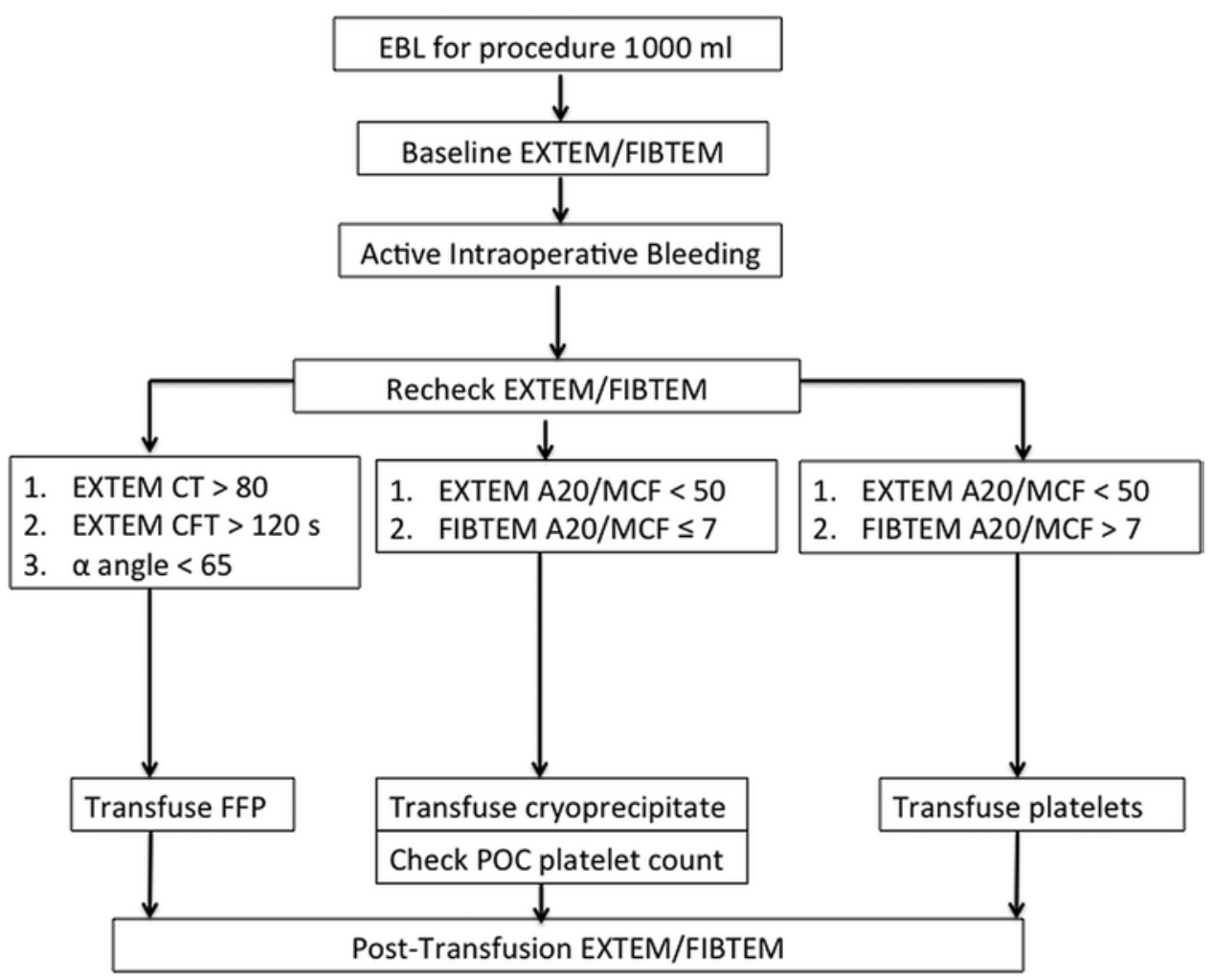

FIG. 2. Transfusion algorithm used in the ROTEM groups (ROTEM-TXA and ROTEM-nonTXA). $\alpha=\alpha$ angle; CFT = clot formation time; $\mathrm{CT}$ = clotting time; $\mathrm{EBL}$ = estimated blood loss; EXTEM = external temogram; FFP = fresh-frozen plasma; FIBTEM = fibrinogen temogram; MCF = maximum clot firmness.

matched at a 1:2 ratio while 4 patients were matched 1:1. The matching cohort was limited to patients who had undergone surgery within the last 5 years to reduce the risk of significant changes in anesthesia and surgical practice. Variable matching in the groups was related to exclusion of potential controls who were inappropriate matches and patients with incomplete perioperative data.

\section{Demographic Data}

Patients in the ROTEM subgroups and their respective controls were well matched for sex, age, ASA physical status, and body mass index (Tables 1 and 2).

\section{Surgical Parameters}

\section{TXA-Treated Patients}

There was no significant difference between the ROTEM-treated and conventionally treated patients with respect to the number of surgically treated vertebral levels (median 9 levels [range 5-18 levels] in the ROTEM-TXA group vs 9 levels [range 7-17 levels] in the ConventionalTXA group, $p=0.3$ ). The proportion of patients who had revision surgery was 54\% in the ROTEM-TXA group and $62 \%$ in the Conventional-TXA group. Pedicle subtraction osteotomy (PSO), vertebral column resection, and interbody procedures were performed in $23 \%(\mathrm{n}=6), 4 \%(\mathrm{n}=$ $1)$, and $65 \%(n=17)$ of the patients in the ROTEM-TXA group, respectively. In the Conventional-TXA group, 19\% $(\mathrm{n}=5), 19 \%(\mathrm{n}=5)$, and $46 \%(\mathrm{n}=12)$ were treated with $\mathrm{PSO}$, vertebral column resection, and interbody proce- dures, respectively. Smith-Petersen osteotomies (SPOs) were performed in $46 \%(\mathrm{n}=12)$ of the patients in the ROTEM-TXA group and $42 \%(n=11)$ of those in the Conventional-TXA group.

\section{Non-TXA-Treated Patients}

The median numbers of surgically treated levels in the ROTEM-nonTXA and Conventional-nonTXA subgroups were 4 (range $2-15$ ) and 4 (range $2-13$ ), respectively ( $\mathrm{p}=$ $0.7)$. Fifty percent of the patients in the ROTEM-nonTXA group and 25\% of those in the Conventional-nonTXA group had revision surgery. PSOs, vertebral column resection, and interbody procedures were performed in $0 \%$ (n $=0), 13 \%(\mathrm{n}=3)$, and $38 \%(\mathrm{n}=9)$ of the patients in the ROTEM-nonTXA group, respectively. In the Conventional-nonTXA group, $7 \%(n=3), 0 \%(n=0)$, and $66 \%$ of the patients $(n=29)$ had PSOs, vertebral column resection, and interbody procedures, respectively. SPOs were performed in $13 \%$ of patients $(\mathrm{n}=3)$ in the ROTEM-nonTXA group and $16 \%(\mathrm{n}=6)$ of those in the Conventional-nonTXA group. There was a trend toward more revision surgery and vertebral column resection in the ROTEM-nonTXA group with more interbody procedures performed in the Conventional-nonTXA group.

\section{Coagulation and Transfusion Parameters TXA-Treated Patients}

The preoperative PT, partial thromboplastin time (PTT), platelet count, and hemoglobin level did not differ significantly between the ROTEM-TXA and Con- 


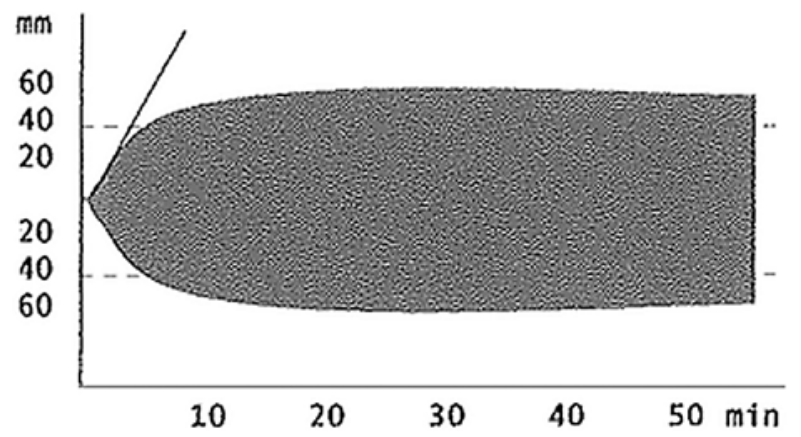

\section{EXTEM}

$\begin{array}{llrrrr}\text { CT } & : & 51 & \text { s [ } & 43- & 82] \\ \text { CFT } & : & 106 & \text { s [ } & 48- & 127] \\ \alpha & : & 73 & \circ[ & 65- & 80] \\ \text { A10 } & : & 53 & \mathrm{~mm} & & \\ \text { A20 } & : & 59 & \mathrm{~mm}[ & 50- & 70] \\ \text { HCF } & : & 60 & \mathrm{~mm}[ & 52- & 70] \\ \text { HL } & : * & 7 & \%[ & 0- & 100] \\ \text { LI } 30 & : & 100 & \% & & \\ \text { LI } 60 & : & & \%\end{array}$

FIG. 3. Example of a normal EXTEM. $A 10=$ amplitude at 10 minutes; $A 20=$ amplitude at 20 minutes; $L I 30=$ lysis index after 30 minutes; LI60 = lysis index after 60 minutes; ML = maximum lysis.

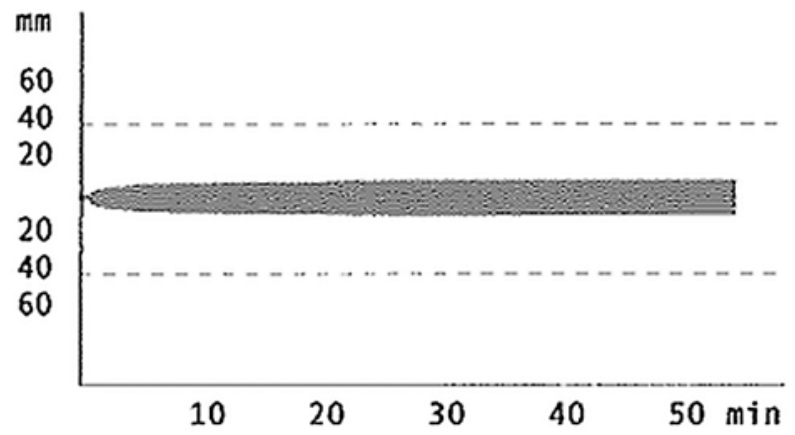

\section{FIBTEM}

\begin{tabular}{|c|c|c|c|c|c|c|c|}
\hline $\mathrm{CT}$ & : & 59 & S & & & & \\
\hline CFT & : & & $S$ & & & & \\
\hline$\alpha$ & : & & 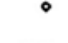 & & & & \\
\hline A10 & : & 8 & $\mathrm{~mm}$ & & & & \\
\hline A20 & : & 9 & $\mathrm{~mm}$ & [ & 7 & - & 24] \\
\hline $\mathrm{MCF}$ & : & 10 & $\mathrm{~mm}$ & [ & 7 & - & 24] \\
\hline ML & $:^{*}$ & 0 & $\%$ & & & & \\
\hline LI30 & : & 100 & $x$ & & & & \\
\hline LI60 & : & & $\%$ & & & & \\
\hline
\end{tabular}

FIG. 4. Example of a normal FIBTEM.

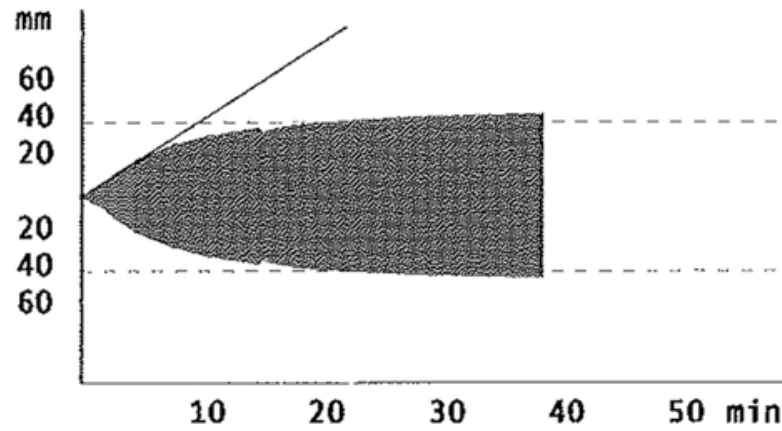

\section{EXTEM}

$\begin{array}{llrrrr}\text { CT } & : & 38 & \text { s [ } & 43- & 82] \\ \text { CFT } & : & 256 & \text { s [ } & 48- & 127] \\ \alpha & : & 47 & \circ[ & 65- & 80] \\ \text { A10 } & : & 32 & \mathrm{~mm} & & \\ \text { A20 } & : & 40 & \mathrm{~mm}[ & 50- & 70] \\ \text { HCF } & : * & 44 & \mathrm{~mm}[ & 52- & 70] \\ \text { HL } & :{ }^{*} & 0 & \%[ & 0- & 100] \\ \text { LI } 30 & : & 100 & \% & & \\ \text { LI } 60 & : & & \% & & \end{array}$

FIG. 5. Example of an EXTEM showing thrombocytopenia.

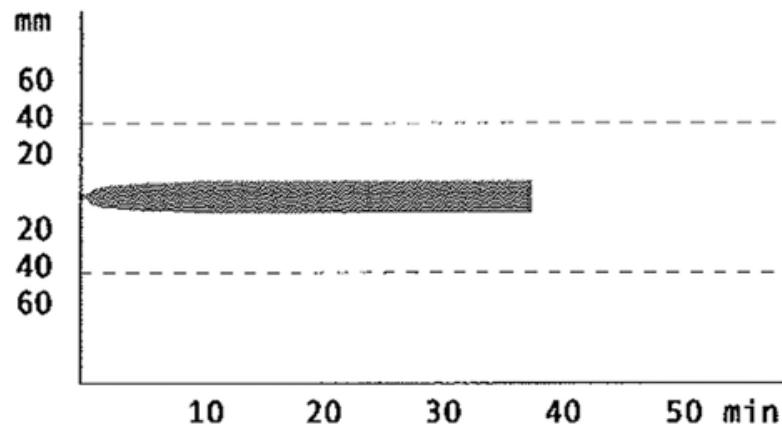

\section{FIBTEM}

$\begin{array}{llrrrrr}\text { CT } & : & 57 & \text { s } & & \\ \text { CFT } & : & & \text { s } & & \\ \alpha & : & & \circ & & \\ \text { A10 } & : & 9 & \text { mm } & & \\ \text { A20 } & : & 9 & \text { mm [ } & 7- & 24] \\ \text { MCF } & : & 9 & \text { mm [ } & 7- & 24] \\ \text { ML } & : * & 3 & \% & & \\ \text { LI30 } & : & 100 & \% & & \\ \text { LI60 } & : & & \% & & \end{array}$

FIG. 6. Example of a FIBTEM showing thrombocytopenia. 


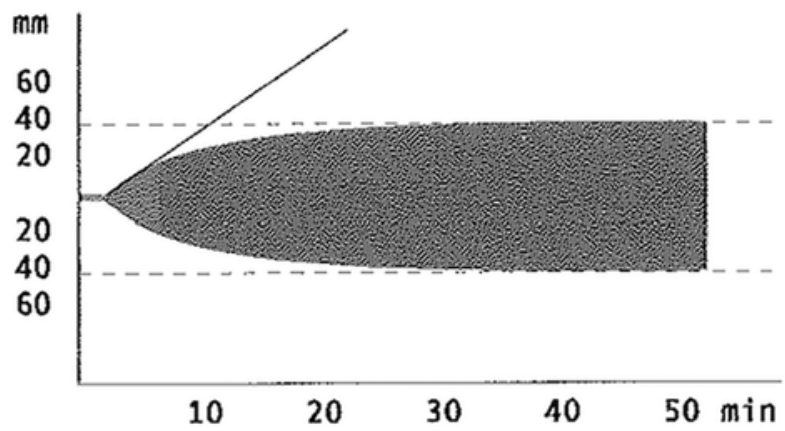

\section{EXTEM}

\begin{tabular}{|c|c|c|c|c|c|}
\hline CT & : & 142 & & 43 & 82] \\
\hline CFT & : & 279 & $\mathbf{s}$ & 48 & - 127] \\
\hline$\alpha$ & : & 49 & 。 & 65 & $80]$ \\
\hline A10 & : & 29 & $\mathrm{~mm}$ & & \\
\hline $\mathrm{A} 20$ & : & 36 & $\mathrm{~mm}$ & 50 & 70] \\
\hline MCF & : & 40 & $\mathrm{~mm}$ & 52 & 70] \\
\hline $\mathrm{HL}$ & $:^{*}$ & 0 & $\%$ & & \\
\hline $\operatorname{Lr} 30$ & : & 100 & \% & & \\
\hline I 60 & & & $\%$ & & \\
\hline
\end{tabular}

FIG. 7. Example of an EXTEM showing hypofibrinogenemia.
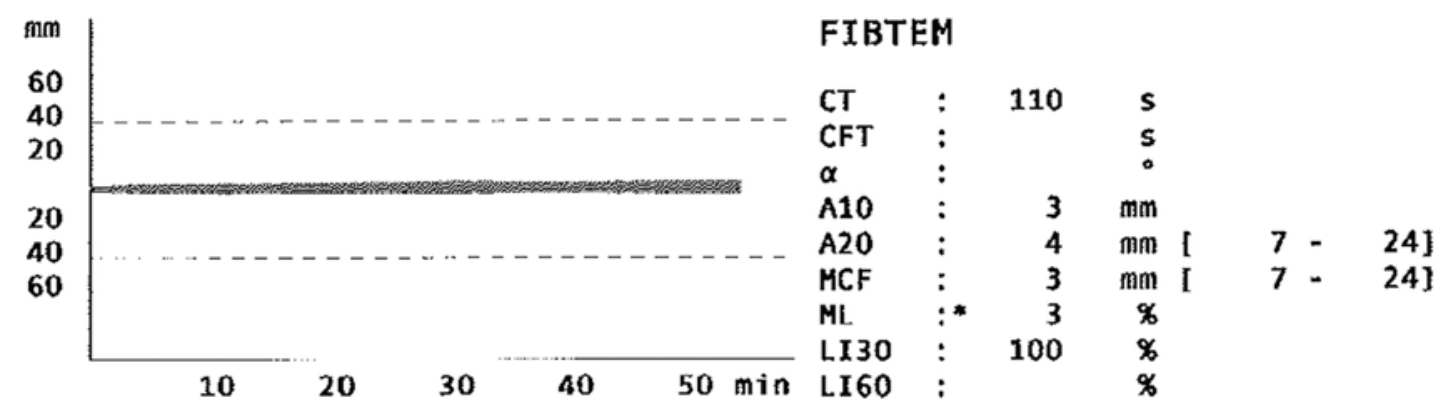

FIG. 8. Example of a FIBTEM showing hypofibrinogenemia.

ventional-TXA groups (Table 1). There was significantly less intraoperative FFP administered with ROTEMguided therapy (median 0 units [range $0-4$ units] for the ROTEM-TXA group vs 2.5 units [range 0-13 units] for the Conventional-TXA group, $\mathrm{p}<0.05$ ), whereas cryoprecipitate use was increased (median 1 unit [range 0-4 units] for the ROTEM-TXA group vs 0 units [range 0-2 units] for the Conventional-TXA group, $p<0.05)$. There was no difference in platelet use between the 2 groups. There was also no significant difference in the quantity of pRBC transfused (median 3 units [range 0-10 units] in the ROTEM-TXA group vs 4 units [range $0-11$ units] in the Conventional-TXA group, $\mathrm{p}=0.1$ ) and blood loss (median $2.6 \mathrm{~L}$ [range $0.9-5.4 \mathrm{~L}$ ] in the ROTEM-TXA group vs 2.9 $\mathrm{L}$ [range $0.7-7.0 \mathrm{~L}$ ] in the Conventional-TXA group, $\mathrm{p}=$ 0.21 ). Autologous blood transfusion volume was significantly lower in the ROTEM group (median $0.8 \mathrm{~L}$ [range $0.3-2.2 \mathrm{~L}]$ in the ROTEM-TXA group vs $1.2 \mathrm{~L}$ [0.47-3.74 $\mathrm{L}]$ in the Conventional-TXA group, $\mathrm{p}=0.02$ ). Postoperative PT, PTT, platelet counts, and blood and blood product use on the day of surgery and on POD 1 did not differ significantly between the 2 groups.

\section{Non-TXA-Treated Patients}

The preoperative coagulation profile did not differ significantly between the ROTEM-nonTXA and Conventional-nonTXA groups (Table 2). With respect to intraoperative blood product administration, the level of use of FFP and platelets was similar in the 2 groups, but more cryoprecipitate was used in the ROTEM-nonTXA subgroup. There was significantly less blood loss (median 1 $\mathrm{L}$ [range $0.2-6.0 \mathrm{~L}$ ] in the ROTEM-nonTXA group vs 1.5 $\mathrm{L}$ [range 1.0-4.5 L] in the Conventional-nonTXA group, $\mathrm{p}$ $<0.05$ ) and less autologous transfusion volume (median 0
$\mathrm{L}$ [range $0-3.8 \mathrm{~L}$ ] in the ROTEM-nonTXA group vs $0.5 \mathrm{~L}$ [range 0-2.2 L] in the Conventional-nonTXA group, $\mathrm{p}=$ $0.05)$. Postoperative values for coagulation parameters did not differ significantly between the 2 groups for measurements obtained on the day of surgery or on POD 1, but there was a trend toward more cryoprecipitate use in the ROTEM-nonTXA group (median 0 units [range 0-2] in the ROTEM-nonTXA group vs 0 units [range $0-1$ unit] in the Conventional-nonTXA group, $p=0.05$ ).

\section{ROTEM Subgroup Analysis}

Patients in the ROTEM-TXA group had more ROTEM testing performed than those in the ROTEM-nonTXA group. In the ROTEM-TXA group, a significant diminution in clot initiation, propagation, and clot strength developed over time, which was not normalized despite transfusion of FFP, cryoprecipitate, and platelets (Table 3). In the ROTEM-nonTXA group, EXTEM $\alpha$ angle, and CFT were the only variables significantly altered from baseline.

\section{Cost Analysis}

If ROTEM-guided protocols were applied in the historical control cases, there could have been cost savings of \$7424 and \$1851 in the Conventional-TXA and Conventional-nonTXA groups, respectively (Table 4). The cost savings were the result of a reduction in pRBC and FFP use in the ROTEM-TXA and ROTEM-nonTXA groups. Cryoprecipitate use increased in both groups to correct the hypofibrinogenemia and reduced clot strength (A10, A20, and MCF) demonstrated on the ROTEM temogram.

\section{Discussion}

The pathogenesis of the coagulopathy encountered in 
TABLE 1. Comparison of perioperative variables between the ROTEM-TXA and Conventional-TXA groups*

\begin{tabular}{|c|c|c|c|}
\hline \multirow[b]{2}{*}{ Parameter } & \multicolumn{2}{|c|}{ Group } & \multirow[b]{2}{*}{ p Value } \\
\hline & ROTEM-TXA & Conventional-TXA & \\
\hline \multicolumn{4}{|l|}{ Baseline demographics } \\
\hline Female sex, \% (n) & $62(16)$ & $58(15)$ & 1 \\
\hline Age in years & $65(45-75)$ & $69(16-80)$ & 0.15 \\
\hline ASA physical status & $2(2-3)$ & $2(1-4)$ & 0.73 \\
\hline Body mass index $\left(\mathrm{kg} / \mathrm{m}^{2}\right)$ & $29(20-46)$ & $28(19-39)$ & 0.27 \\
\hline \multicolumn{4}{|l|}{ Preop lab results } \\
\hline INR & $1(0.8-1.3)$ & $1(0.9-1.4)$ & 0.36 \\
\hline PTT (seconds) & $30(25-47)$ & $29(27-54)$ & 0.35 \\
\hline Hemoglobin (g/dl) & $13(9.8-15.9)$ & $12(8.8-16.7)$ & 0.27 \\
\hline Platelet count $(/ \mu l)$ & $242(122-473)$ & $238(121-393)$ & 0.59 \\
\hline \multicolumn{4}{|l|}{ Intraop surgical variables } \\
\hline No. of spine levels & $9(5-18)$ & $9(7-17)$ & 0.33 \\
\hline Revision surgery, \% (n) & $54(14)$ & $62(16)$ & 0.78 \\
\hline $\mathrm{SPO}, \%(\mathrm{n})$ & $46(12)$ & $42(11)$ & 0.79 \\
\hline PSO, $\%$ (n) & $23(6)$ & $19(5)$ & 1 \\
\hline Interbody procedure, $\%$ (n) & $65(17)$ & $46(12)$ & 0.26 \\
\hline Vertebral column resection, $\%$ (n) & $4(1)$ & $19(5)$ & 0.19 \\
\hline \multicolumn{4}{|l|}{ Blood, blood products, \& fluids } \\
\hline pRBC (units) & $3(0-10)$ & $4(0-11)$ & 0.1 \\
\hline FFP (units) & $0(0-4)$ & $2.5(0-13)$ & 0.0002 \\
\hline Cryoprecipitate (units) & $1(0-4)$ & $0(0-2)$ & 0.005 \\
\hline Platelet (units) & $0(0-1)$ & $0(0-2)$ & 0.3 \\
\hline Autologous blood transfusion (L) & $0.8(0.3-2.2)$ & $1.2(0.47-3.74)$ & 0.02 \\
\hline Blood loss (L) & $2.6(0.9-5.4)$ & $2.9(0.7-7.0)$ & 0.21 \\
\hline Crystalloid (L) & $3.3(2.0-5.5)$ & $2.5(1.0-6.2)$ & 0.03 \\
\hline Colloid (L) & $1.5(0-2.5)$ & $1.5(0-2.8)$ & 0.5 \\
\hline TXA (mg) & $1261(732-1954)$ & $1030(651-3000)$ & 0.44 \\
\hline \multicolumn{4}{|l|}{ Postop lab results, day of surgery } \\
\hline INR & $1.3(1.2-1.7)$ & $1.3(1.1-1.9)$ & 0.53 \\
\hline PTT (seconds) & $28(22-38)$ & $27(21-47)$ & 0.5 \\
\hline Platelets $(/ \mu \mathrm{l})$ & $131(75-283)$ & $126(42-285)$ & 0.7 \\
\hline Hemoglobin (g/dl) & $10.3(7-13.2)$ & $10.2(6.9-11.8)$ & 0.4 \\
\hline \multicolumn{4}{|l|}{ POD 1 lab results } \\
\hline INR & $1.3(1.1-1.9)$ & $1.3(1.1-1.4)$ & 0.3 \\
\hline PTT (seconds) & $26(20-33)$ & $25(23-85)$ & 0.7 \\
\hline Platelet count $(/ \mu \mathrm{l})$ & $118(37-269)$ & $140(92-263)$ & 0.2 \\
\hline Hemoglobin (g/dl) & $10(7-13.2)$ & $10.6(8.4-12.7)$ & 0.8 \\
\hline \multicolumn{4}{|c|}{ Blood \& blood products transfused w/in 24 hrs postop } \\
\hline pRBC (units) & $1(0-5)$ & $0(0-2)$ & 0.06 \\
\hline FFP (units) & $0(0-5)$ & $0(0-2)$ & 0.06 \\
\hline Platelet (units) & $0(0-2)$ & $0(0-2)$ & 0.08 \\
\hline Cryoprecipitate (units) & $0(0-1)$ & $0(0-1)$ & 1 \\
\hline
\end{tabular}

* Data are presented as medians and ranges unless otherwise indicated.

major spine surgery is multifold and includes hypothermia, dilution of coagulation factors, hypofibrinogenemia, and activation of the fibrinolytic system through bonemediated tissue plasminogen activator and urokinase. ${ }^{3,8,15}$ In a study by Murray et al., significantly elevated PT and PTT and a nonsignificant trend toward lower fibrinogen levels and platelet counts were seen in 32 patients undergoing posterior spinal fusion. ${ }^{15}$ In contrast, Carling et al. reported that total bleeding volume was significantly correlated only with preoperative fibrinogen concentration and not PT, PTT, or platelet counts in patients undergoing correction of idiopathic scoliosis. ${ }^{2}$ Standard and POC test- 
TABLE 2. Comparison of perioperative variables between the ROTEM-nonTXA and Conventional-nonTXA groups*

\begin{tabular}{|c|c|c|c|}
\hline \multirow[b]{2}{*}{ Parameter } & \multicolumn{2}{|c|}{ Group } & \multirow[b]{2}{*}{ p Value } \\
\hline & ROTEM-nonTXA & Conventional-nonTXA & \\
\hline \multicolumn{4}{|l|}{ Baseline demographics } \\
\hline Female sex \% (n) & $33(8)$ & $52(23)$ & 0.2 \\
\hline Age in yrs & $67(49-79)$ & $62(24-74)$ & 0.02 \\
\hline ASA physical status & $2(2-4)$ & $2(2-4)$ & 0.9 \\
\hline Body mass index $\left(\mathrm{kg} / \mathrm{m}^{2}\right)$ & $30(21-56)$ & $33(20-46)$ & 0.58 \\
\hline \multicolumn{4}{|l|}{ Preop lab results } \\
\hline INR & $1(0.9-1.4)$ & $1(0.8-1.2)$ & 0.07 \\
\hline PTT (seconds) & $30(27-37)$ & $30(24-41)$ & 0.9 \\
\hline Hemoglobin (g/dl) & $13(7.4-15.3)$ & $14(9-16.8)$ & 0.59 \\
\hline Platelet count $(/ \mu \mathrm{L})$ & $224(129-459)$ & $214(135-695)$ & 0.96 \\
\hline \multicolumn{4}{|l|}{ Intraop surgical variables } \\
\hline Spine levels & $4(3-15)$ & $4(3-15)$ & 0.77 \\
\hline Revision surgery, \% (n) & $50(12)$ & $25(11)$ & 0.06 \\
\hline $\mathrm{SPO}, \%$ (n) & $13(3)$ & $16(6)$ & 1 \\
\hline PSO, \% (n) & $0(0)$ & $7(3)$ & 0.55 \\
\hline Interbody procedure, \% (n) & $38(9)$ & $66(29)$ & 0.04 \\
\hline Vertebral column resection, \% (n) & $13(3)$ & $0(0)$ & 0.04 \\
\hline \multicolumn{4}{|l|}{ Blood, blood products, \& fluids } \\
\hline pRBC (units) & $0(0-4)$ & $1(0-9)$ & 0.09 \\
\hline FFP (units) & $0(0-5)$ & $0(0-7)$ & 0.6 \\
\hline Cryoprecipitate (units) & $0(0-3)$ & $0(0)$ & 0.02 \\
\hline Platelets (units) & $0(0-1)$ & $0(0-1)$ & 0.4 \\
\hline Autologous blood transfusion (L) & $0(0-3.8)$ & $0.5(0-2.2)$ & 0.05 \\
\hline Blood loss (ml) & $1(0.2-6.0)$ & $1.5(1.0-4.5)$ & 0.0005 \\
\hline Crystalloid (ml) & $2.8(0.7-6.0)$ & $3.9(1.4-9.5)$ & 0.0008 \\
\hline Colloid (ml) & $0.6(0-4.0)$ & $1.0(0-3.5)$ & 0.3 \\
\hline \multicolumn{4}{|l|}{ Postop lab results, day of surgery } \\
\hline INR & $1.2(1-1.8)$ & $1.2(1.1-2)$ & 0.5 \\
\hline PTT (seconds) & $28(25-35)$ & $28(22-38)$ & 1 \\
\hline Platelets $(/ \mu \mathrm{L})$ & $177(68-329)$ & $157(79-311)$ & 0.4 \\
\hline Hemoglobin (g/dl) & $11(9-13.2)$ & $10.8(8.4-15.3)$ & 0.5 \\
\hline \multicolumn{4}{|l|}{ POD 1 lab results } \\
\hline INR & $1.2(1-1.6)$ & $1.2(1.1-2.7)$ & 0.4 \\
\hline PTT (seconds) & $26(22-35)$ & $26(23-92)$ & 0.9 \\
\hline Platelets $(/ \mu \mathrm{L})$ & $147(76-316)$ & $148(52-268)$ & 1 \\
\hline Hemoglobin (g/dl) & $10.3(8.5-12.4)$ & $10.7(7.9-12.9)$ & 0.2 \\
\hline \multicolumn{4}{|c|}{ Blood \& blood products transfused w/in 24 hrs postop } \\
\hline pRBC (units) & $0(0-2)$ & $0(0-3)$ & 0.5 \\
\hline FFP (units) & $0(0-2)$ & $0(0-5)$ & 0.06 \\
\hline Platelets (units) & $0(0-2)$ & $0(0-2)$ & 0.6 \\
\hline Cryoprecipitate (units) & $0(0-2)$ & $0(0-1)$ & 0.05 \\
\hline
\end{tabular}

* Data are presented as medians and ranges unless otherwise indicated.

ing may not be accurate during major blood loss such as traumatic coagulopathy. Davenport et al. reported median laboratory reporting times of 78 minutes (range 62-103 minutes) for PT, with a $29 \%$ false-negative rate for POC PT values. ${ }^{4}$

ROTEM offers a rapid real-time assessment of the coagulation pathway. ROTEM is a viscoelastometric he- mostasis test that utilizes whole blood. Blood is placed in a cuvette within which a cylindrical pin is immersed. ${ }^{11}$ There is a 1-mm distance between the pin and cuvette, which is bridged by blood. When the pin is initially rotated after blood is added to the cuvette, there is minimal resistance to the rotation. With increasing clot formation, there is increased resistance to rotation, which is detected by the 
TABLE 3. Intraoperative changes in EXTEM and FIBTEM variables*

\begin{tabular}{|c|c|c|c|c|c|}
\hline \multirow[b]{2}{*}{ Variable } & \multicolumn{4}{|c|}{ Assay } & \multirow[b]{2}{*}{$\mathrm{p}$ Value } \\
\hline & Baseline & $1 \mathrm{st}$ & 2nd & $3 \mathrm{rd}$ & \\
\hline \multicolumn{6}{|l|}{ ROTEM-TXA group } \\
\hline EXTEM CT (seconds) & $48 \pm 2$ & $63 \pm 4$ & $62 \pm 4$ & $55 \pm 8$ & 0.014 \\
\hline EXTEM CFT (seconds) & $96 \pm 7$ & $133 \pm 10$ & $144 \pm 11$ & $176 \pm 20$ & $<0.001$ \\
\hline EXTEM a $\left(^{\circ}\right)$ & $72 \pm 1$ & $66 \pm 2$ & $64 \pm 2$ & $59 \pm 3$ & $<0.001$ \\
\hline EXTEM A10 (mm) & $54 \pm 1$ & $47 \pm 2$ & $45 \pm 2$ & $41 \pm 2$ & $<0.001$ \\
\hline EXTEM A20 (mm) & $61 \pm 1$ & $55 \pm 2$ & $52 \pm 2$ & $39 \pm 2$ & $<0.001$ \\
\hline EXTEM MCF (mm) & $62 \pm 1$ & $56 \pm 2$ & $54 \pm 2$ & $51 \pm 2$ & 0.002 \\
\hline EXTEM ML (\%) & $6 \pm 1$ & $6 \pm 2$ & $4 \pm 1$ & $5 \pm 1$ & $<0.001$ \\
\hline FIBTEM A20 (mm) & $17 \pm 1$ & $12 \pm 1$ & $10 \pm 1$ & $8 \pm 2$ & 0.005 \\
\hline FIBTEM MCF (mm) & $17 \pm 1$ & $13 \pm 2$ & $10 \pm 2$ & $8 \pm 2$ & 0.005 \\
\hline \multicolumn{6}{|l|}{ ROTEM-nonTXA group } \\
\hline EXTEM CT (seconds) & $53 \pm 4$ & $56 \pm 4$ & & & 0.626 \\
\hline EXTEM CFT (seconds) & $95 \pm 6$ & $131 \pm 15$ & & & 0.013 \\
\hline EXTEM $\alpha\left({ }^{\circ}\right)$ & $72 \pm 1$ & $65 \pm 3$ & & & 0.005 \\
\hline EXTEM A10 (mm) & $54 \pm 2$ & $48 \pm 3$ & & & 0.085 \\
\hline EXTEM A20 (mm) & $60 \pm 1$ & $55 \pm 3$ & & & 0.063 \\
\hline EXTEM MCF (mm) & $61 \pm 1$ & $57 \pm 3$ & & & 0.090 \\
\hline EXTEM ML (\%) & $5 \pm 1$ & $7 \pm 3$ & & & 0.620 \\
\hline FIBTEM A20 (mm) & $16 \pm 1$ & $12 \pm 2$ & & & 0.122 \\
\hline FIBTEM MCF (mm) & $16 \pm 1$ & $13 \pm 2$ & & & 0.125 \\
\hline
\end{tabular}

* Data are presented as mean \pm standard error; $p$ values are based on 1-way ANOVA.

optical sensor and reported as both a functional parameter and a representative curve. To mimic traditional coagulation pathways, different reagents are added to the cuvette to initiate the hemostatic process. For the extrinsic pathway, calcium and tissue factor are used (EXTEM assay). Adding a reagent with direct platelet inhibition activity, such as cytochalasin, produces a representative temogram of fibrin formation and polymerization (FIBTEM assay). The technical aspects of ROTEM are summarized in a review by Keene et al. ${ }^{11}$

TABLE 4. Predicted cost savings with ROTEM-guided therapy

\begin{tabular}{lrrr}
\hline Blood Product & $\begin{array}{c}\text { Actual Cost in } \\
\text { Conventional } \\
\text { Group }\end{array}$ & $\begin{array}{c}\text { Predicted Cost if } \\
\text { ROTEM Were Used } \\
\text { in Conventional } \\
\text { Group }\end{array}$ & Difference \\
\hline TXA-treated patients & & & \\
\hline pRBC & $\$ 25,650$ & $\$ 20,025$ & $-\$ 5625$ \\
\hline FFP & $\$ 2175$ & $\$ 400$ & $-\$ 775$ \\
\hline Cryoprecipitate & $\$ 558$ & $\$ 1674$ & $+\$ 1116$ \\
\hline Platelets & $\$ 4815$ & $\$ 2675$ & $-\$ 2140$ \\
\hline Total cost & $\$ 33,198$ & $\$ 25,774$ & $-\$ 7424$ \\
\hline Non-TXA-treated patients & & & \\
\hline pRBC & $\$ 10,330$ & $\$ 9900$ & $-\$ 430$ \\
\hline FFP & $\$ 1344$ & $\$ 896$ & $-\$ 448$ \\
\hline Cryoprecipitate & $\$ 0$ & $\$ 682$ & $+\$ 682$ \\
\hline Platelets & $\$ 2675$ & $\$ 980$ & $-\$ 1695$ \\
\hline Total cost & $\$ 14,309$ & $\$ 12,458$ & $-\$ 1851$ \\
\hline
\end{tabular}

The aim of this study was to determine whether applying an allogeneic product transfusion strategy based on ROTEM in complex multilevel spine surgery is associated with decreased blood and blood component therapy use. Our study supports the usefulness of the technique by reporting lower pRBC and FFP usage in cases in which ROTEM therapy was used, even in cases in which it was used in conjunction with antifibrinolytic therapy. In cases in which ROTEM-guided therapy was used without antifibrinolytic therapy with TXA, allogeneic product saving was limited to $\mathrm{pRBC}$ only. This dichotomous effect is most likely related to the selection of TXA for more complex spine cases, for which the anticipated blood loss is large. This is supported by the median number of surgically treated vertebral levels (median 9 for TXA-treated patients vs 4 for non-TXA-treated patients) and blood loss in the TXA-treated patients (median 2.6 L in the ROTEMTXA group and $2.9 \mathrm{~L}$ in the Conventional-TXA group) compared with the non-TXA-treated patients $(1 \mathrm{~L}$ in the ROTEM-nonTXA group and $1.5 \mathrm{~L}$ in the ConventionalnonTXA group). In the ROTEM-nonTXA group, bleeding, coagulopathy, and transfusion therapy were limited, mitigating the beneficial effects of ROTEM-guided therapy. Furthermore, our cost analysis demonstrated significant health care dollar savings when ROTEM is applied in this manner (Table 4). In centers performing a high volume of spine procedures with large amounts of blood loss, the potential cost savings from allogeneic transfusions are likely to outweigh the capital and consumables cost of ROTEM. Spalding et al., in an analysis of pre- and post-ROTEM costs of cardiac surgery, demonstrated a $32 \%(\$ 28,000 /$ 
month) reduction in all blood product use with a monthly ROTEM cost of $\$ 2166 .{ }^{20}$

In our study the reduction in blood and component therapy intraoperatively was not associated with any detrimental effects in the immediate postoperative coagulation profile. Hemoglobin level, PT, PTT, and platelet counts on admission to the inpatient floor after surgery did not differ significantly between the patients in the ROTEM and Conventional groups. Among TXA-treated patients, there was a trend toward slightly more pRBC and FFP use within the first 24 hours in the ROTEM-TXA group than in the Conventional-TXA group (pRBC: median 1 unit [range $0-5$ units] vs 0 units [range $0-2$ units], respectively, $\mathrm{p}=$ 0.06 ; FFP: median 0 units [range $0-5$ ] vs 0 units [range $0-2$ units], $p=0.06$ ). Among patients who did not receive TXA, there was a trend toward more cryoprecipitate use in the ROTEM-nonTXA group than in the ConventionalnonTXA group (1 unit [range $0-2$ units] vs 0 units [range $0-1$ units], $p=0.05$ ). Increased transfusion with nonsignificant differences in coagulation parameters is likely reflective of nonstandardized postoperative transfusion triggers at our institution.

Finally, an important observation from our study was the difference in blood loss between the ROTEM-TXA and the Conventional-TXA groups (median 2.6 L [range $0.9-5.4 \mathrm{~L}$ ] vs $2.9 \mathrm{~L}$ [range $0.7-7.0 \mathrm{~L}$ ], $\mathrm{p}=0.21$ ) and between the ROTEM-nonTXA and Conventional-nonTXA groups (median $1 \mathrm{~L}$ [range $0.2-6.0 \mathrm{~L}$ ] vs $1.5 \mathrm{~L}$ [range 1.0-4.5 L], $\mathrm{p}=0.0005$ ), with greater blood loss in the patients without ROTEM-guided therapy despite no significant differences in the postoperative coagulation profiles of the patient groups. These results highlight the limitations of the INR, PTT, and quantitative platelet count with respect to the assessment of functional in vivo clotting and planning of transfusion treatment, while supporting the use of thromboelastogram values, contrary to the findings of Horlocker et al. ${ }^{8}$

Avoiding unnecessary allogeneic transfusions reduces the risk of transfusion-related complications. The risk of death associated with a transfusion is approximately $1: 322,580$, while the risk of major morbidity is $1: 21,413 .{ }^{1}$ The most common causes of death associated with an allogeneic transfusion are transfusion-related acute lung injury, followed by hemolytic transfusion reactions. The risk of transfusion-related acute lung injury is estimated to be between $0.08 \%$ and $15 \%$ following a transfusion. ${ }^{22}$ Risk factors for developing transfusion-related acute lung injury include mechanical ventilation, massive transfusion, and increased plasma use, which was significantly lower in the patients treated with the ROTEM-guided approach. ${ }^{21,22}$ These factors are common denominators in major spine surgery.

Analysis of the different coagulation pathways by ROTEM in our study highlights the importance of functional hypofibrinogenemia in major spine surgery. A significant reduction of the MCF in the EXTEM and FIBTEM from baseline-indicative of a reduction in qualitative fibrinogen and/or platelet function-is evident (Table 3). The normal postsurgery platelet counts in our study point to the important contribution of low fibrinogen to the reduced MCF seen on the EXTEM. The FIBTEM MCF has been shown to correlate well with laboratory fibrinogen values, with a FIBTEM MCF of less than $7 \mathrm{~mm}$, approximating $150 \mathrm{mg} / \mathrm{dl}$ of plasma fibrinogen. ${ }^{19}$ In trauma-induced coagulopathy, a FIBTEM MCF of less than $7 \mathrm{~mm}$ is associated with profuse bleeding. ${ }^{11} \mathrm{FFP}$ is a poor choice for the correction of hypofibrinogenemia, as it has a low concentration of fibrinogen compared with cryoprecipitate or fibrinogen concentrate. Standard preparations of FFP and cryoprecipitate have approximately $2 \mathrm{~g} / \mathrm{L}(0.6 \mathrm{~g}$ in a typical $300-$ $\mathrm{ml} \mathrm{bag}$ ) and $15 \mathrm{~g} / \mathrm{L}$ of fibrinogen. ${ }^{7}$ In our study the mean intraoperative fibrinogen values in the ROTEM-TXA and Conventional-TXA groups were $258 \pm 164 \mathrm{mg} / \mathrm{dl}$ and 175 $\pm 37 \mathrm{mg} / \mathrm{dl}$, respectively, as a result of more cryoprecipitate use in the ROTEM-TXA group.

Fibrinogen concentrate is a purified concentrate of fibrinogen derived from human plasma using the Cohn/Oncley cryoprecipitation procedure. ${ }^{7}$ Each ampule provides 1 $\mathrm{g} / \mathrm{L}$ of fibrinogen, which is reconstituted in $50 \mathrm{ml}$ of water. Therefore, the same amount of fibrinogen as FFP can be administered in one-sixth of the volume. Although primarily indicated for prophylactic and therapeutic treatment of hemorrhagic diathesis with congenital afibrinogenemia or hypofibrinogenemia, there are studies reporting its use in acquired hypofibrinogemia during major blood loss surgical procedures. ${ }^{6,10}$ Mittermayr et al. reported improved ROTEM clot polymerization and strength with the use of fibrinogen concentrate in patients undergoing spine surgery (involving more than 3 segments) with a dilutional coagulopathy. ${ }^{14}$ In the US, fibrinogen concentrate (Haemocomplettan P 1g, CSL Behring) is approved by the FDA only for the treatment of acute bleeding episodes in patients with congenital fibrinogen deficiency (afibrinogenemia or hypofibrinogenemia).

Multiple studies have demonstrated reduced blood loss with prophylactic antifibrinolytic use in spine surgery. ${ }^{13,26}$ However, in our study there was no evidence of hyperfibrinolysis (EXTEM ML > 15\%) in the ROTEM-nonTXA group. A limited number of ROTEM temograms were performed in this group, as a result of the lower blood loss, and late fibrinolysis could potentially have been missed.

Limitations of our study include a small sample size with potential variability in transfusion practice in the historical controls due to varying anesthesia providers. We attempted to limit this variability by restricting the matching period to a 5-year period so that significant changes in transfusion practices and staffing were reduced. Furthermore, there was no standardized surgical measure to quantify the degree of spine surgery being performed. Matching based on the number of surgically treated levels may result in inappropriate matching.

\section{Conclusions}

We have demonstrated that the application of a goaldirected hemostatic approach with functional coagulation testing increases cryoprecipitate but reduces FFP use. This is due to hypofibrinogenemia as a major contributor to the ongoing coagulopathy. Early identification and management of hypofibrinogenemia is critical in major spine surgery as demonstrated by a reduction in blood loss with cryoprecipitate use. ROTEM offers a new, safe, and 
cost-effective method to allogeneic blood product management in major spine surgery. Furthermore, by applying a ROTEM-guided algorithm, variance of transfusion practice can be reduced with increased standardization of care among different providers.

\section{References}

1. Bolton-Maggs PH, Cohen H: Serious Hazards of Transfusion (SHOT) haemovigilance and progress is improving transfusion safety. Br J Haematol 163:303-314, 2013

2. Carling MS, Jeppsson A, Wessberg P, Henriksson A, Baghaei F, Brisby H: Preoperative fibrinogen plasma concentration is associated with perioperative bleeding and transfusion requirements in scoliosis surgery. Spine (Phila Pa 1976) 36:549-555, 2011

3. Dahl OE, Pedersen T, Kierulf P, Westvik AB, Lund P, Arnesen $\mathrm{H}$, et al: Sequential intrapulmonary and systemic activation of coagulation and fibrinolysis during and after total hip replacement surgery. Thromb Res 70:451-458, 1993

4. Davenport R, Manson J, De'Ath H, Platton S, Coates A, Allard S, et al: Functional definition and characterization of acute traumatic coagulopathy. Crit Care Med 39:26522658,2011

5. Dirkmann D, Görlinger K, Dusse F, Kottenberg E, Peters J: Early thromboelastometric variables reliably predict maximum clot firmness in patients undergoing cardiac surgery: a step towards earlier decision making. Acta Anaesthesiol Scand 57:594-603, 2013

6. Fenger-Eriksen C, Jensen TM, Kristensen BS, Jensen KM, Tønnesen E, Ingerslev J, et al: Fibrinogen substitution improves whole blood clot firmness after dilution with hydroxyethyl starch in bleeding patients undergoing radical cystectomy: a randomized, placebo-controlled clinical trial. J Thromb Haemost 7:795-802, 2009

7. Franchini M, Lippi G: Fibrinogen replacement therapy: a critical review of the literature. Blood Transfus 10:23-27, 2012

8. Horlocker TT, Nuttall GA, Dekutoski MB, Bryant SC: The accuracy of coagulation tests during spinal fusion and instrumentation. Anesth Analg 93:33-38, 2001

9. Hu SS: Blood loss in adult spinal surgery. Eur Spine J 13 (Suppl 1):S3-S5, 2004

10. Karlsson M, Ternström L, Hyllner M, Baghaei F, Flinck A, Skrtic S, et al: Prophylactic fibrinogen infusion reduces bleeding after coronary artery bypass surgery. A prospective randomised pilot study. Thromb Haemost 102:137-144, 2009

11. Keene DD, Nordmann GR, Woolley T: Rotational thromboelastometry-guided trauma resuscitation. Curr Opin Crit Care 19:605-612, 2013

12. Kelly MP, Lenke LG, Shaffrey CI, Ames CP, Carreon LY, Lafage V, et al: Evaluation of complications and neurological deficits with three-column spine reconstructions for complex spinal deformity: a retrospective Scoli-RISK-1 study. Neurosurg Focus 36(5):E17, 2014

13. Li ZJ, Fu X, Xing D, Zhang HF, Zang JC, Ma XL: Is tranexamic acid effective and safe in spinal surgery? A meta-analysis of randomized controlled trials. Eur Spine J 22:1950-1957, 2013

14. Mittermayr M, Streif W, Haas T, Fries D, Velik-Salchner C, Klingler A, et al: Hemostatic changes after crystalloid or colloid fluid administration during major orthopedic sur- gery: the role of fibrinogen administration. Anesth Analg 105:905-917, 2007

15. Murray DJ, Pennell BJ, Weinstein SL, Olson JD: Packed red cells in acute blood loss: dilutional coagulopathy as a cause of surgical bleeding. Anesth Analg 80:336-342, 1995

16. Naik BI, Colquhoun DA, McKinney WE, Smith AB, Titus B, McMurry TL, et al: Incidence and risk factors for acute kidney injury after spine surgery using the RIFLE classification. J Neurosurg Spine 20:505-511, 2014

17. Norton RP, Bianco K, Lafage V, Schwab FJ: Complications and intercenter variability of three-column resection osteotomies for spinal deformity surgery: a retrospective review of 423 patients. Evid Based Spine Care J 4:157-159, 2013

18. Ogawa S, Szlam F, Chen EP, Nishimura T, Kim H, Roback JD, et al: A comparative evaluation of rotation thromboelastometry and standard coagulation tests in hemodilutioninduced coagulation changes after cardiac surgery. Transfusion 52:14-22, 2012

19. Rugeri L, Levrat A, David JS, Delecroix E, Floccard B, Gros A, et al: Diagnosis of early coagulation abnormalities in trauma patients by rotation thrombelastography. J Thromb Haemost 5:289-295, 2007

20. Spalding GJ, Hartrumpf M, Sierig T, Oesberg N, Kirschke CG, Albes JM: Cost reduction of perioperative coagulation management in cardiac surgery: value of "bedside" thrombelastography (ROTEM). Eur J Cardiothorac Surg 31:10521057, 2007

21. Vlaar AP, Binnekade JM, Prins D, van Stein D, Hofstra JJ, Schultz MJ, et al: Risk factors and outcome of transfusionrelated acute lung injury in the critically ill: a nested casecontrol study. Crit Care Med 38:771-778, 2010

22. Vlaar AP, Juffermans NP: Transfusion-related acute lung injury: a clinical review. Lancet 382:984-994, 2013

23. Weber CF, Görlinger K, Meininger D, Herrmann E, Bingold T, Moritz A, et al: Point-of-care testing: a prospective, randomized clinical trial of efficacy in coagulopathic cardiac surgery patients. Anesthesiology 117:531-547, 2012

24. Weiss G, Lison S, Spannagl M, Heindl B: Expressiveness of global coagulation parameters in dilutional coagulopathy. $\mathbf{B r}$ J Anaesth 105:429-436, 2010

25. Whitaker BI, Hinkins S: The 2011 National Blood Collection and Utilization Survey Report. Rockville, MD: US Department of Health and Human Services, 2011

26. Wong J, El Beheiry H, Rampersaud YR, Lewis S, Ahn H, De Silva Y, et al: Tranexamic Acid reduces perioperative blood loss in adult patients having spinal fusion surgery. Anesth Analg 107:1479-1486, 2008

\section{Author Contributions}

Conception and design: Naik, Durieux, Shaffrey, Nemergut. Acquisition of data: Naik. Analysis and interpretation of data: Naik, Nemergut. Drafting the article: Naik. Critically revising the article: Naik, Pajewski, Bogdonoff, Zuo, Clark, Durieux, Shaffrey, Nemergut. Reviewed submitted version of manuscript: Naik. Approved the final version of the manuscript on behalf of all authors: Naik. Statistical analysis: Naik, Terkawi. Administrative/ technical/material support: Naik. Study supervision: Naik.

\section{Correspondence}

Bhiken I. Naik, Department of Anesthesiology, University of Virginia, P.O. Box 800710, Charlottesville, VA 22908-0710. email: bin4n@virginia.edu. 